Marquette University

e-Publications@Marquette

Exercise Science Faculty Research and

Publications

Exercise Science, Department of

$2-2018$

\title{
Validation of the Godin Leisure-Time Exercise Questionnaire Classification Coding System Using Accelerometry in Multiple Sclerosis
}

Robert W. Motl

University of Alabama, Birmingham

Rachel Bollaert

Marquette University, rachel.bollaert@marquette.edu

Brian M. Sandroff

University of Alabama - Birmingham

Follow this and additional works at: https://epublications.marquette.edu/exsci_fac

\section{Recommended Citation}

Motl, Robert W.; Bollaert, Rachel; and Sandroff, Brian M., "Validation of the Godin Leisure-Time Exercise Questionnaire Classification Coding System Using Accelerometry in Multiple Sclerosis" (2018). Exercise Science Faculty Research and Publications. 162.

https://epublications.marquette.edu/exsci_fac/162 
Marquette University

e-Publications@Marquette

\title{
Exercise Science Faculty Research and Publications/College of Health Sciences
}

This paper is NOT THE PUBLISHED VERSION; but the author's final, peer-reviewed manuscript. The published version may be accessed by following the link in the citation below.

Rehabilitation Psychology, Vol. 63, No. 1 (February 2018): 77-82. DOI. This article is (C) American Psychological Association and permission has been granted for this version to appear in ePublications@Marquette. American Psychological Association does not grant permission for this article to be further copied/distributed or hosted elsewhere without the express permission from American Psychological Association.

\section{Validation of the Godin Leisure-Time Exercise Questionnaire Classification Coding System Using Accelerometry in Multiple Sclerosis}

\author{
Robert W. Motl
}

Department of Physical Therapy, School of Health Professions, University of Alabama at Birmingham; Rachel E. Bollaert Department of Kinesiology and Community Health, University of Illinois at Urbana-Champaign Brian M. Sandroff

Department of Physical Therapy, School of Health Professions, University of Alabama at Birmingham

\footnotetext{
Abstract

Objective: There have been recent efforts toward creating a health contribution score (HCS) from the Godin Leisure-Time Exercise Questionnaire (GLTEQ) that reflects public-health guidelines for levels of moderate-tovigorous physical activity (MVPA). The HCS yields categories of insufficiently active (less substantial or low
} 
benefits), moderately active (some benefits), and active (substantial benefits). The present study examined the validity of the GLTEQ HCS and its categories as reflecting levels of MVPA in multiple sclerosis (MS). Method: The sample included 684 persons with MS. Participants wore an accelerometer on an elastic belt around the waist above the nondominant hip during the waking hours of the day over a 7-day period and completed the GLTEQ. Results: The data analyses supported a large correlation between the GLTEQ HCS and accelerometer-measured MVPA, $r=.46, p<.0001$, but small correlations with accelerometer-measured light physical activity (LPA), $r=$ $.16, p<.001$ and sedentary time, $r=-.13, p=.001$. There further was a large difference in accelerometermeasured MVPA between categories of physical activity levels (i.e., insufficiently active vs. active) based on the GLTEQ HCS $(d=0.89)$, but small differences in LPA $(d=0.39)$ and sedentary time $(d=-0.31)$. Those results were unchanged in additional data analyses accounting for LPA and sedentary behavior. Conclusions: The GLTEQ HCS and categories primarily reflect MVPA rather than LPA and sedentary behavior in persons with MS. (PsycINFO Database Record (c) 2018 APA, all rights reserved)

\section{Impact Statement}

Impact and Implications - We validated the health contribution score from the Godin Leisure-Time Exercise Questionnaire as reflecting public-health guidelines for levels of moderate-to-vigorous physical activity in persons with multiple sclerosis. This is important for describing samples and/or categorizing mean scores and changes over time with an intervention. This is relevant for identifying patients who might require exercise promotion and/or rehabilitation programs for targeting comorbid conditions

\section{Introduction}

There is increasing interest by researchers and clinicians in understanding and promoting physical activity behavior among persons with MS (Lai, Young, Bickel, Motl, \& Rimmer, 2017; Motl, Learmonth, Pilutti, Gappmaier, \& Coote, 2015). The existing research supports that engaging in physical activity has substantial benefit for persons with MS (Motl \& Sandroff, 2015), and the benefits may be even larger when meeting public health guidelines for physical activity behavior (Adamson, Ensari, \& Motl, 2015). Public health guidelines for physical activity reflect the amount of MVPA necessary for reducing the risks of morbidity and mortality as well as optimizing the likelihood of experiencing cardiorespiratory, musculoskeletal, and neuromotor benefits of this behavior (Garber et al., 2011). The existence of public health guidelines, in part, provide an MVPA benchmark for health care providers and policymakers when making recommendations on health promoting physical activity levels among adults 18-64 years of age, including adults with disabilities such as MS.

The increasing interest by researchers and clinicians in understanding and promoting physical activity behavior among persons with MS requires measures with scores that yield valid and reliable inferences (i.e., score meaning). The measures should further provide scores that can be easily interpreted and translatable within the context of public health guidelines for physical activity. This latter quality of a measure is important in research for describing samples and/or categorizing mean scores and changes over time with an intervention. This further is important in clinical practice for identifying patients who might require promotion and/or rehabilitation programs for targeting comorbid conditions.

The GLTEQ (Godin \& Shephard, 1985) might fulfill the aforementioned qualities for application in MS. The GLTEQ is one of the most commonly adopted scales for measuring physical activity in MS (Gosney, Scott, Snook, \& Motl, 2007; Kinnett-Hopkins, Adamson, Rougeau, \& Motl, 2017), and contains three core items regarding the frequency of strenuous, moderate, and mild physical activity for bouts of 15 or more minutes during a 7-day period. The scores are multiplied by weights and summed into an overall score (i.e., leisure-time physical activity [LTPA] score) that ranges between 0 and 119 metabolic equivalents of task (METs)/minutes of physical activity 
per week. There is evidence for the reliability of LTPA scores from the GLTEQ over a 6-month period in persons with MS (Motl, McAuley, \& Klaren, 2014). There is additional evidence for the validity of LTPA scores in MS based on correlations with other self-report measures of physical activity as well as objective markers of physical activity from accelerometry (Gosney et al., 2007). We further note that LTPA scores have captured the efficacy of behavioral interventions for increasing physical activity in MS (Pilutti, Dlugonski, Sandroff, Klaren, \& Motl, 2014). However, the LTPA score does not directly reflect public-health guidelines for levels of physical activity (i.e., Garber et al., 2011) for ease of interpretation among researchers and clinicians.

There have been recent efforts toward creating a HCS from the GLTEQ that reflects public-health guidelines for levels of physical activity (Amireault \& Godin, 2015; Godin, 2011). The HCS is computed using only the sum of the weighted strenuous and moderate scores from the GLTEQ, and scores reflect categories of insufficiently active (less than 14 units; less substantial or low benefits), moderately active (14-23 units; some benefits), and active (24 units or more; substantial benefits). We are unaware of research validating the GLTEQ HCS and its categories as reflecting levels and differences in MVPA among persons with MS. This is an important precursor for the application and interpretation of the GLTEQ HCS in clinical research and practice involving persons with MS.

We are aware of one previous study examining the validity of the GLTEQ HCS for classifying breast cancer survivors into active and insufficiently active categories (Amireault, Godin, Lacombe, \& Sabiston, 2015). That study included 199 breast cancer survivors who wore an accelerometer on the waist during the waking hours of the day for seven consecutive days and completed the GLTEQ after the 7-day period. There was a moderate sized correlation between accelerometer-measured MVPA minutes/week and the GLTEQ HCS ( $r=.46)$, and a large $(d=.78)$ difference between active and insufficiently active HCS groups/categories in accelerometermeasured MVPA minutes/week. Such data support the convergent validity of the HCS score as reflecting MVPA in a clinical population, but do not provide divergent validity regarding smaller correlations with other measures of behavior, including LPA and sedentary behavior. This is important for identifying that the HCS score captures MVPA rather than LPA and sedentary behavior.

The present study validated the GLTEQ HCS and its categories as reflecting levels of MVPA in MS. We first examined the correlation between HCS and minutes per day of MVPA, LPA, and sedentary behavior measured by waist-worn accelerometry, and then examined if the categories of physical activity based on the GLTEQ HCS reflected differences in amounts of MVPA, LPA, and sedentary behavior. We opted for accelerometry as another "method" of measuring physical activity for construct validity, consistent with the frameworks of Cronbach and Meehl (1955) and Campbell and Fiske (1959), and note that it provides an objective measure of ambulatory physical activity with metrics (i.e., cut points) that have been calibrated with indirect calorimetry while walking in MS (Sandroff, Motl, \& Suh, 2012). We hypothesized (a) a large correlation between the HCS and minutes/day of MVPA (i.e., $r \sim$.50), but a small correlation between the HCS and minutes/day of LPA and sedentary behavior (i.e., $r \sim$.10), and (b) large differences in minutes/day of MVPA between categories of physical activity levels (i.e., insufficiently active vs. active) based on HCS (i.e., $d \sim .80$ ), but small HCS group differences in LPA and sedentary behavior (i.e., $d \sim$.30). If our hypotheses are supported, this study would provide convergent and divergent evidence for the construct validity of the GLTEQ HCS and its categories in MS.

\section{Method}

\section{Sample}

Prospective participants were recruited from the Midwestern portion of the United States using multiple sources, including print and e-mail flyers, an online advertisement on the National Multiple Sclerosis Society website, and our database of previous participants with MS. The advertisements described a study examining 
physical activity behavior in persons with MS. The inclusion criteria were (a) definite physician diagnosis of MS that was confirmed in writing by the participant's physician, (b) self-reported relapse-free in the previous 30 days, (c) ambulatory with or without assistive devices, and (d) age between 18 and 64 years. The final sample included 684 persons with MS who volunteered and completed the study procedures.

\section{Measures}

\section{GLTEQ}

The GLTEQ measures the frequency of strenuous, moderate, and mild LTPA performed for periods of 15 min or more over a usual week. Recently, a new scoring method has been proposed for generating an overall GLTEQ score that aligns with current recommendations for physical activity and the dose-response association between the volume of physical activity and health benefits (i.e., HCS; Amireault \& Godin, 2015; Godin, 2011). The HCS is based on only strenuous and moderate physical activity and is computed by multiplying the frequencies of strenuous and moderate activities by 9 and $5 \mathrm{METs}$, respectively, and then adding the resultant scores. The HCS ranges between 0 and 98 and is converted into one of three categories, namely, insufficiently active (i.e., score $<14$ units that is the equivalent of $<7 \mathrm{kcal} / \mathrm{kg} / \mathrm{week}$ ), moderately active (i.e., score between 14 and 23 units that is the equivalent of between 7 and $13.9 \mathrm{kcal} / \mathrm{kg} /$ week), and active (i.e., score $\geq 24$ units that exceeds 13.9 $\mathrm{kcal} / \mathrm{kg} /$ week).

\section{Accelerometry}

The study included a pool of 100 ActiGraph Model 7164 accelerometers, as this model and its location of placement (i.e., waist) has been validated for capturing intra- and interperson variation in ambulatory physical activity in MS (Motl, Sosnoff, Dlugonski, Suh, \& Goldman, 2010). This model of accelerometer measures physical activity as activity counts per unit time using a piezoelectric bender element that produces an electrical signal proportionate to the force acting on it during movement. The electric signal is digitally converted into activity counts amalgamated over 1-min sampling intervals (i.e., 60-s epochs), and the activity counts per minute are stored in the accelerometer's random access memory. Of note, all 100 accelerometers were calibrated prior to data collection for accurately measuring physical activity by laboratory staff members who walked on a treadmill $(4.8 \mathrm{~km} / \mathrm{h}, 0 \%$ grade for $15 \mathrm{~min}$ ) while wearing the accelerometers on a belt around the waist. Accuracy was confirmed by a $<10 \%$ difference in average counts per minute across the 15 -min period of walking among the accelerometers worn simultaneously. The data from the accelerometers were downloaded and processed using Actilife software. The data were then processed into two separate Microsoft Excel files representing wear time and time spent in sedentary behavior, LPA, and MVPA; this later processing was based on activity count cut points for persons with MS (i.e., sedentary: 0-99 counts/minute; LPA: 100-1,722 counts/minute; MVPA: $\geq 1,723$ counts/minute; Sandroff, Motl, \& Suh, 2012). Those cut points were derived from a direct calibration of accelerometer output based on its strong association with energy expenditure during walking in a sample of 43 persons with MS who ambulated with or without assistive devices $\left(R^{2}=0.91\right.$; Sandroff et al., 2012). Accelerometer wear time data were checked against participant recorded wear times from a log sheet, and only valid days (>10 hr of wear time without periods of continuous zeros exceeding 60 min indicative of noncompliance) were included in the analyses. All participants had two or more days of valid accelerometer data, regardless of weekday or weekend day (Motl et al., 2007), and we averaged the available and valid daily data into a metric of average minutes/day for MVPA, LPA, and sedentary behavior.

\section{Patient Determined Disease Steps (PDDS) Scale}

The PDDS provides a patient-reported measure of disability status in MS, and has nine ordinal levels ranging between 0 (normal) and 8 (bedridden; Hadjimichael, Kerns, Rizzo, Cutter, \& Vollmer, 2007). The scores from this scale have been validated in MS based on correlations with scores from the Expanded Disability Status Scale $(r=$ 
.783) as well as measures of ambulation including the 6-min walk $(r=-.704)$, timed 25 -foot walk $(r=.627)$, and Multiple Sclerosis Walking Scale-12 ( $r=.800$; Learmonth, Motl, Sandroff, Pula, \& Cadavid, 2013).

\section{Procedures}

The procedures were approved by a university institutional review board, and all participants provided informed consent. Participants were screened over the telephone for inclusion criteria, and those who qualified received initial instructions on the study procedures, including properly wearing the accelerometer. We then sent participants a packet through the United States Postal Service containing the accelerometer along with a log sheet and standardized instructions with pictures, the GLTEQ, Demographic/Clinical Questionnaire, PDDS, and a prestamped/preaddressed envelope for returning the materials. We further phoned participants for ensuring understanding with instructions for wearing the accelerometer and completing the GLTEQ. We instructed the participants to wear the accelerometer around the waist above the nondominant hip using the provided elastic belt during the waking hours of the day over a 7-day period and recorded wear time in a log sheet. Waking hours was defined as the time from waking and getting out of bed in the morning until getting into bed for sleep in the evening. Participants completed the GLTEQ within $24 \mathrm{hr}$ after wearing the accelerometer, and completed the Demographic/Clinical Characteristic Questionnaire and PDDS for characterizing the sample when convenient. The materials were returned through the United States Postal Service in a prestamped and preaddressed envelope. We paid participants $\$ 25$ for completing the study procedures.

\section{Data Analysis}

The data were analyzed in IBM SPSS Version 24.0. We provide descriptive statistics as mean score and standard deviation $(S D)$, unless otherwise noted. The first primary analysis examined the association between the GLTEQ HCS and minutes/day of MVPA, LPA, and sedentary behavior from the accelerometer using a Pearson productmoment correlation coefficient $(r)$, and interpreted the magnitude based on guidelines of $.1, .3$, and .5 as small, moderate, and large, respectively (Cohen, 1988). The second primary analysis compared minutes/day of MVPA as well as LPA and sedentary behavior from the accelerometer among the three categories of physical activity based on the GLTEQ HCS using a one-way analysis of variance (ANOVA) with Bonferroni adjusted post hoc analyses ( $p=.0167$ ) per ANOVA (i.e., controlling error rate per analysis), and interpreted the magnitude of group differences based on guidelines of .3, .5, and .8 as small, moderate, and large, respectively (Cohen, 1988). We further report partial eta-squared $\left(\eta^{2}\right)$ as an overall effect size estimate per ANOVA with values of .01, .06, and .12 representing small, medium, and large effects, respectively (Cohen, 1988).

\section{Results}

\section{Sample Characteristics}

The sample of 684 persons with MS had a mean (SD) age of 47.1 (10.2) years, and consisted of 554 women and 102 men; 28 persons did not report sex. The sample mean (SD) disease duration was 9.8 (7.5) years, and 598 had relapsing-remitting MS (RRMS) and 71 had progressive MS; 15 persons had an unknown disease course. The median (interquartile range) PDDS score was 2.0 (3.0) indicating moderate ambulatory disability. There were 176 persons (25.6\%) who used an assistive device for ambulation based on the criterion of a PDDS score of 4.0 (i.e., early cane) or greater.

\section{Physical Activity Profile}

LPA and sedentary behavior scores approximated a normal distribution, whereas HCS and MVPA scores deviated from a normal distribution (i.e., positive skewness); this is consistent with the distribution of MVPA in MS and the general population of adults (i.e., very few persons are currently achieving recommended levels of physical activity; Kinnett-Hopkins et al., 2017). The distribution of HCS scores is provided in Figure 1, and the skewness and kurtosis values for the distribution were 1.45 and 1.85 , respectively. We included parametric analyses 
considering robustness against deviations from normality with such a large sample size (Tabachnik \& Fidell, 1996).

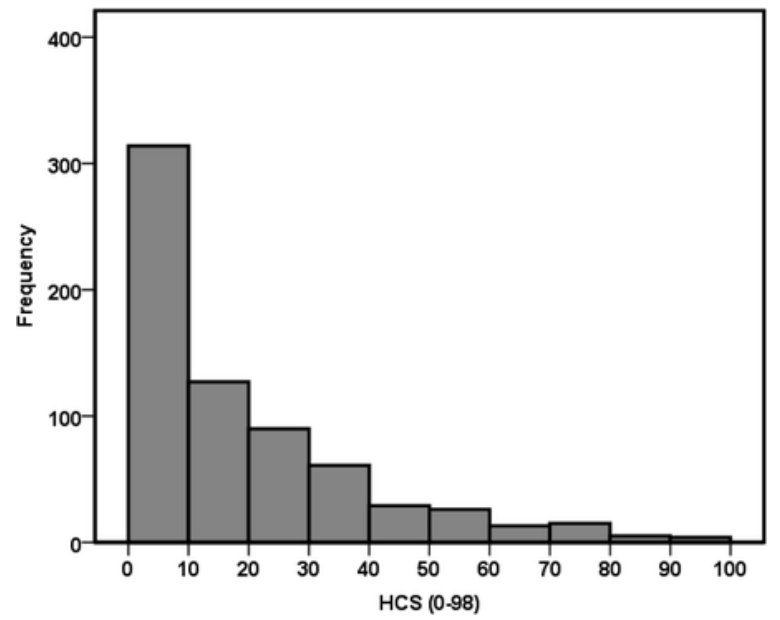

Figure 1. Histogram of health composite scores (HCS) from the Godin Leisure Time Exercise Questionnaire in 684 persons with multiple sclerosis.

The mean (SD) for the GLTEQ HCS was 16.8 (20.6). Based on the GLTEQ HCS, there were 367 (53.7\%), 109 (15.9\%), and 208 (30.4\%) cases of persons with MS in the insufficiently active, moderately active, and active groups, respectively. The mean (SD) scores for minutes/day of MVPA, LPA, and sedentary time from the accelerometer were 19.3 (19.6), 290.2 (79.3), and 526.1 (93.0), respectively. The mean (SD) wear time was 835.1 (80.0) minutes/day.

\section{Correlation Between Scores}

There was a statistically significant and strong correlation between the GLTEQ HCS and accelerometer-measured minutes/day of MVPA, $r=.46, p<.0001$. That correlation was significantly larger in magnitude than the correlations between the HCS and accelerometer-measured minutes/day of LPA, $r=.16, p<.001$ and sedentary behavior, $r=-.13, p=.001$, based on Fisher's $z$ test for comparing the different in dependent correlations (Steiger, 1980). Importantly, the correlation between the GLTEQ HCS and accelerometer-measured minutes/day of MVPA was virtually unchanged when controlling for LPA and sedentary time in a follow-up partial correlation analysis $(p r=.44, p<.0001)$.

\section{Comparison of Scores Among Groups}

The mean (SD) minutes/day of MVPA, LPA, and sedentary behavior from the accelerometer for the three groups of insufficiently active, moderately active, and active are provided in Table 1 . The ANOVA indicated an overall large and statistically significant difference among groups $\left(F(2,681)=50.8, p<.0001, \eta^{2}=.13\right)$, and follow-up comparisons with Bonferroni correction indicated that all three groups differed significantly in minutes/day of MVPA. The effect sizes for insufficiently active versus moderately active groups and moderately active versus active groups were 0.34 and 0.51 , respectively. The effect size for insufficiently active versus active groups was 0.89 . 
Table 1

Descriptive Statistics for Physical Activity and Sedentary Outcomes From the Accelerometer Across Groups of Physical Activity Based on the Health Composite Score From the Godin Leisure Time Exercise Questionnaire

\begin{tabular}{lccr}
\hline & \multicolumn{2}{c}{ Health composite score group } & \\
\cline { 2 - 3 } Accelerometer variable & Insufficiently active & Moderately active & Active \\
\hline MVPA (minutes/day) & $13.6(14.5)$ & $18.7(16.1)$ & $29.6(24.3)$ \\
LPA (minutes/day) & $280.3(78.4)$ & $285.6(82.8)$ & $310.1(75.6)$ \\
Sedentary (minutes/day) & $534.0(93.4)$ & $538.3(95.8)$ & $505.6(87.8)$ \\
\hline
\end{tabular}

Note. MVPA $=$ moderate-to-vigorous physical activity; $\mathrm{LPA}=$ light physical activity.

Table 1 Descriptive Statistics for Physical Activity and Sedentary Outcomes From the Accelerometer Across Groups of Physical Activity Based on the Health Composite Score From the Godin Leisure Time Exercise Questionnaire

\begin{tabular}{|l|l|l|l|}
\hline & $\begin{array}{l}\text { Health composite score } \\
\text { group }\end{array}$ & & \\
\hline Accelerometer variable & Insufficiently active & Moderately active & Active \\
\hline MVPA (minutes/day) & $13.6(14.5)$ & $18.7(16.1)$ & $29.6(24.3)$ \\
\hline LPA (minutes/day) & $280.3(78.4)$ & $285.6(82.8)$ & $310.1(75.6)$ \\
\hline Sedentary (minutes/day) & $534.0(93.4)$ & $538.3(95.8)$ & $505.6(87.8)$ \\
\hline
\end{tabular}

The ANOVA indicated an overall small, but statistically significant, difference among groups $(F(2,681)=9.9, p<$ $\left..0001, \eta^{2}=.03\right)$, and follow-up comparisons with Bonferroni correction indicated that the inactive and insufficiently active groups both differed significantly from the active group in minutes/day of LPA. The effect sizes for insufficiently active versus moderately active groups and moderately active versus active groups were 0.07 and 0.31 , respectively. The effect size for insufficiently active versus active groups was 0.39 .

The ANOVA indicated an overall small, but statistically significant, difference among groups $(F(2,681)=7.5, p<$ $\left..001, \eta^{2}=.02\right)$, and follow-up comparisons with Bonferroni correction indicated that the inactive and insufficiently active groups both differed significantly from the active group in minutes/day of sedentary behavior. The effect sizes for insufficiently active versus moderately active groups and moderately active versus active groups were 0.04 and -0.36 , respectively. The effect size for insufficiently active versus active groups was -0.31 .

\section{Independence of Group Differences in MVPA}

We conducted an analysis of covariance (ANCOVA) for comparing groups on accelerometer-measured minutes/day of MVPA controlling for LPA and sedentary time, and there was an overall moderate-to-large and statistically significant difference among groups $\left(F(2,679)=42.8, p<.0001, \eta^{2}=.09\right)$. The follow-up comparisons with Bonferroni correction indicated that all three groups based on the GLTEQ HCS differed significantly in accelerometer-measured minutes/day of MVPA. The adjusted mean minutes/day of MVPA from the accelerometer were $14.0,19.2$, and 28.6 for insufficiently active, moderately active, and active groups, respectively.

\section{Discussion}

This study examined the validity of the GLTEQ HCS and its categories (Amireault \& Godin, 2015; Godin, 2011) as a measure of MVPA in persons with MS. The notable results, as expected, supported (a) a large correlation between the GLTEQ HCS and accelerometer-measured MVPA, but small correlations between the GLTEQ HCS and accelerometer-measured LPA and sedentary behavior; (b) a large difference in accelerometer-measured 
MVPA between categories of physical activity levels (i.e., insufficiently active vs. active) based on the GLTEQ HCS, but small differences in LPA and sedentary behavior; and (c) independence of both the correlation between the HCS and accelerometer-measured MVPA and the difference in accelerometer-measured MVPA across HCS categories when accounting for LPA and sedentary behavior. This pattern of results suggests that the GLTEQ HCS and categories primarily reflect MVPA (i.e., convergent validity) rather than LPA and sedentary behavior (i.e., divergent validity) in persons with MS. These results may have significant value for researchers and clinicians who are interested in understanding and promoting physical activity behavior among persons with MS. We have provided evidence that the GLTEQ HCS can be interpreted regarding public health guidelines for physical activity. This is important as researchers can adopt the GLTEQ HCS for describing samples and/or categorizing mean scores and changes over time with an intervention. We further believe that there is relevance for clinical practice wherein health care providers can apply the GLTEQ HCS for identifying patients who might require physical activity promotion and/or rehabilitation programs for targeting comorbid conditions. This is important, given that the existence of public health guidelines, in part, provide a basis for health care providers and policymakers when making recommendations on health-promoting physical activity levels among adults 18-64 years of age, including adults with disabilities such as MS.

Our results are consistent with previous research examining the validity of the GLTEQ HCS using accelerometry in a sample of 199 breast cancer survivors (Amireault et al., 2015). That study reported a similar sized correlation $(r=.46)$ between accelerometer-measured MVPA minutes/week and the GLTEQ HCS, and a large $(d=.78)$ difference between active and insufficient active groups/categories in MVPA minutes/week. One important distinction between studies is that our analyses controlled for LPA and sedentary behavior, and reported independence of the association between HCS and minutes/day of MVPA. Collectively, these results confirm that the GLTEQ HCS can be interpreted as reflecting MVPA in breast cancer survivors and persons with MS, and our results further strengthen this inference as unique for MVPA rather than LPA and sedentary behavior based on additional partial correlation and ANCOVA analyses in a large sample of persons with MS. This latter point is important, as the HCS score only includes moderate and strenuous physical activity and excludes LPA.

We envision several next steps as well as implications for future research and practice based on the results of this study. Regarding next steps, researchers might consider examining the usefulness of the GLTEQ HCS for predicting short- and long-term health outcomes such as depression, fatigue, pain, and quality of life in MS. Researchers might further consider examining the usefulness of the GLTEQ HCS as a potential consequence of disease-related outcomes (i.e., mobility disability). This, in turn, might provide a guideline for prescreening subjects based on inclusion/exclusion for randomized controlled trials of physical activity behavior change in MS. We might further examine the utility of the GLTEQ HCS in clinical practice for making recommendations on health behavior change and/or referrals for counseling on behavior change. The GLTEQ HCS is a relatively simple scoring algorithm on a straightforward questionnaire that is easily interpretable for both practitioners and patients within the context of published public health guidelines for accruing benefits associated with MVPA behavior. As such, the GLTEQ HCS might be particularly advantageous for use in such clinical settings, considering the specialized equipment, software, and expertise required for objective accelerometry.

There are some limitations of this study. The first limitation is that the accelerometer for validating the HCS was worn on a belt around the waist, and this captures only ambulatory physical activity. To that end, the correlations and differences we reported might have been underestimated by missing water-based physical activity and/or cycling, as examples, although we note that walking is the most common type of physical activity undertaken in MS (Weikert, Dlugonski, Balantrapu, \& Motl, 2011). Another limitation was that we only included cross-sectional data and analyses, and did not provide evidence for the test-retest reliability of the HCS over time or changes in the HCS and its categories as reflecting changes in MVPA over time. The last limitation was 
that the sample primarily included women with RRMS who were ambulatory, thereby limiting generalizability among the broader community with MS.

Overall, we provide the first evidence for the validity of inferences from the GLTEQ HCS and its categories as reflecting MVPA in MS, and this evidence is complementary with that provided in breast cancer survivors (Amireault et al., 2015). There is room for additional investigation regarding the validity and value of GLTEQ HCS in $\mathrm{MS}$, but the current evidence is strong enough for recommendations that researchers and clinicians can adopt it in clinical research and practice involving MS. Such application has obvious value for describing samples and/or categorizing mean scores and changes over time in clinical research, and identifying patients who might require promotion and/or rehabilitation programs for targeting comorbid conditions in clinical practice.

\section{References}

Adamson, B. C., Ensari, I., \& Motl, R. W. (2015). Effect of exercise on depressive symptoms in adults with neurological disorders: A systematic review an meta-analysis. Archives of Physical Medicine and Rehabilitation, 96, 1329-1338. 10.1016/j.apmr.2015.01.005

Amireault, S., \& Godin, G. (2015). The Godin-Shephard Leisure-Time Physical Activity Questionnaire: Validity evidence supporting its use for classifying healthy adults into active and insufficiently active categories. Perceptual and Motor Skills, 120, 604-622. 10.2466/03.27.PMS.120v19x7

Amireault, S., Godin, G., Lacombe, J., \& Sabiston, C. M. (2015). Validation of the Godin-Shephard Leisure-Time Physical Activity Questionnaire classification coding system using accelerometer assessment among breast cancer survivors. Journal of Cancer Survivorship, 9, 532-540. 10.1007/s11764-015-0430-6

Campbell, D. T., \& Fiske, D. W. (1959). Convergent and discriminant validation by the multitrait-multimethod matrix. Psychological Bulletin, 56, 81-105. 10.1037/h0046016

Cohen, J. (1988). Statistical power analysis for the behavioral sciences (2nd ed.). Hillsdale, NJ: Erlbaum.

Cronbach, L. J., \& Meehl, P. E. (1955). Construct validity in psychological tests. Psychological Bulletin, 52, 281302. 10.1037/h0040957

Garber, C. E., Blissmer, B., Deschenes, M. R., Franklin, B. A., Lamonte, M. J., Lee, I. M., . . the American College of Sports Medicine. (2011). American College of Sports Medicine position stand. Quantity and quality of exercise for developing and maintaining cardiorespiratory, musculoskeletal, and neuromotor fitness in apparently healthy adults: Guidance for prescribing exercise. Medicine and Science in Sports and Exercise, 43, 1334-1359. 10.1249/MSS.0b013e318213fefb

Godin, G. (2011). The Godin-Shephard Leisure-Time Physical Activity Questionnaire. Health and Fitness Journal of Canada, 4, 18-22.

Godin, G., \& Shephard, R. J. (1985). A simple method to assess exercise behavior in the community. Canadian Journal of Applied Sports Sciences, 10, 141-146.

Gosney, J. L., Scott, J. A., Snook, E. M., \& Motl, R. W. (2007). Physical activity and multiple sclerosis: Validity of self-report and objective measures. Family \& Community Health, 30, 144-150. 10.1097/01.FCH.0000264411.20766.0c

Hadjimichael, O., Kerns, R. D., Rizzo, M. A., Cutter, G., \& Vollmer, T. (2007). Persistent pain and uncomfortable sensations in persons with multiple sclerosis. Pain, 127, 35-41. 10.1016/j.pain.2006.07.015

Kinnett-Hopkins, D., Adamson, B., Rougeau, K., \& Motl, R. W. (2017). People with MS are less physically active than healthy controls but as active as those with other chronic diseases: An updated metaanalysis. Multiple Sclerosis and Related Disorders, 13, 38-43. 10.1016/j.msard.2017.01.016

Lai, B., Young, H. J., Bickel, C. S., Motl, R. W., \& Rimmer, J. H. (2017). Current trends in exercise intervention research, technology, and behavioral change strategies for people with disability: A scoping review. American Journal of Physical Medicine \& Rehabilitation. Advance online publication. 10.1097/phm.0000000000000743

Learmonth, Y. C., Motl, R. W., Sandroff, B. M., Pula, J. H., \& Cadavid, D. (2013). Validation of patient determined disease steps (PDDS) scale scores in persons with multiple sclerosis. BMC Neurology, 13, 37. 10.1186/1471-2377-13-37 
Motl, R. W., Learmonth, Y. C., Pilutti, L. A., Gappmaier, E., \& Coote, S. (2015). Top 10 research questions related to physical activity and multiple sclerosis. Research Quarterly for Exercise and Sport, 86, 117-129. 10.1080/02701367.2015.1023099

Motl, R. W., McAuley, E., \& Klaren, R. (2014). Reliability of physical-activity measures over six months in adults with multiple sclerosis: Implications for designing behavioral interventions. Behavioral Medicine, 40, 2933. $10.1080 / 08964289.2013 .821966$

Motl, R. W., \& Sandroff, B. M. (2015). Benefits of exercise training in multiple sclerosis. Current Neurology and Neuroscience Reports, 15, 62. 10.1007/s11910-015-0585-6

Motl, R. W., Sosnoff, J. J., Dlugonski, D., Suh, Y., \& Goldman, M. (2010). Does a waist-worn accelerometer capture intra- and inter-person variation in walking behavior among persons with multiple sclerosis?Medical Engineering \& Physics, 32, 1224-1228. 10.1016/j.medengphy.2010.08.015

Motl, R. W., Zhu, W., Park, Y., McAuley, E., Scott, J. A., \& Snook, E. M. (2007). Reliability of scores from physical activity monitors in adults with multiple sclerosis. Adapted Physical Activity Quarterly, 24, 245-253. 10.1123/apaq.24.3.245

Pilutti, L. A., Dlugonski, D., Sandroff, B. M., Klaren, R., \& Motl, R. W. (2014). Randomized controlled trial of a behavioral intervention targeting symptoms and physical activity in multiple sclerosis. Multiple Sclerosis Journal, 20, 594-601. 10.1177/1352458513503391

Sandroff, B. M., Motl, R. W., \& Suh, Y. (2012). Accelerometer output and its association with energy expenditure in persons with multiple sclerosis. Journal of Rehabilitation Research and Development, 49, 467-475. 10.1682/JRRD.2011.03.0063

Steiger, J. H. (1980). Tests for comparing elements of a correlation matrix. Psychological Bulletin, 87, 245-251. 10.1037/0033-2909.87.2.245

Tabachnick, B. G., \& Fidell, L. S. (1996). Using multivariate statistics. New York, NY: HarperCollins College.

Weikert, M., Dlugonski, D., Balantrapu, S., \& Motl, R. W. (2011). Most common types of physical activity selfselected by people with multiple sclerosis. International Journal of MS Care, 13, 16-20. 10.7224/15372073-13.1.16 